\title{
Optimal Time Intervals for Vaginal Breech Births: A Case-Control Study
}

\author{
Emma Spillane ${ }^{1}$, Shawn Walker ${ }^{2}$, and Christine McCourt ${ }^{3}$ \\ ${ }^{1}$ Kingston Hospital NHS Foundation Trust \\ ${ }^{2}$ King's College London \\ ${ }^{3}$ City University London
}

September 24, 2021

\begin{abstract}
Objective: To test the predictive value of the Physiological Breech Birth Algorithm. Design: Retrospective case-control study Setting: Teaching Hospital, United Kingdom Population/sample: Cases were all vaginal breech births $>37$ weeks' gestation where neonatal admission or death occurred between April 2012 and April 2020. Controls were the two term breech births without admission immediately prior to the cases. Methods: Data was collected from intrapartum care records and analysed using SPSS v26 statistical software. The chi-square test was used to determine association between exposure to the variables of interest and admission to the neonatal unit. Multiple logistic regression was used to test the predictive value of delays defined as non-adherence to the Algorithm. Main outcome measures: Intervals between the start of labour, the start of second stage of labour and various stages of emergence (presenting part, buttocks, pelvis, arms, head). Results: Logistic regressing modelling using the Algorithm time frames had an $84.2 \%$ accuracy, a sensitivity of $66.7 \%$ and a specificity of $92.3 \%$. Delays between umbilicus and head $>3$ minutes (OR: 9.508 [95\% CI: 1.390-65.046] p=0.022) and from buttocks on the perineum to head $>7$ minutes (OR: 6.682 [95\% CI: 0.940-41.990] p=0.058) showed the most effect. Lengths of time until the first intervention were also longer among the cases, suggesting that at least some of this delay is modifiable. Conclusions: Improved recognition of delay and efficient assistance may help improve vaginal breech birth outcomes. Further research should determine whether training based on the Physiological Breech Birth Algorithm can reduce neonatal admissions.
\end{abstract}

\section{Hosted file}

BJOG Optimal timings 2 Aug.docx available at https://authorea.com/users/435262/articles/ 538412-optimal-time-intervals-for-vaginal-breech-births-a-case-control-study 

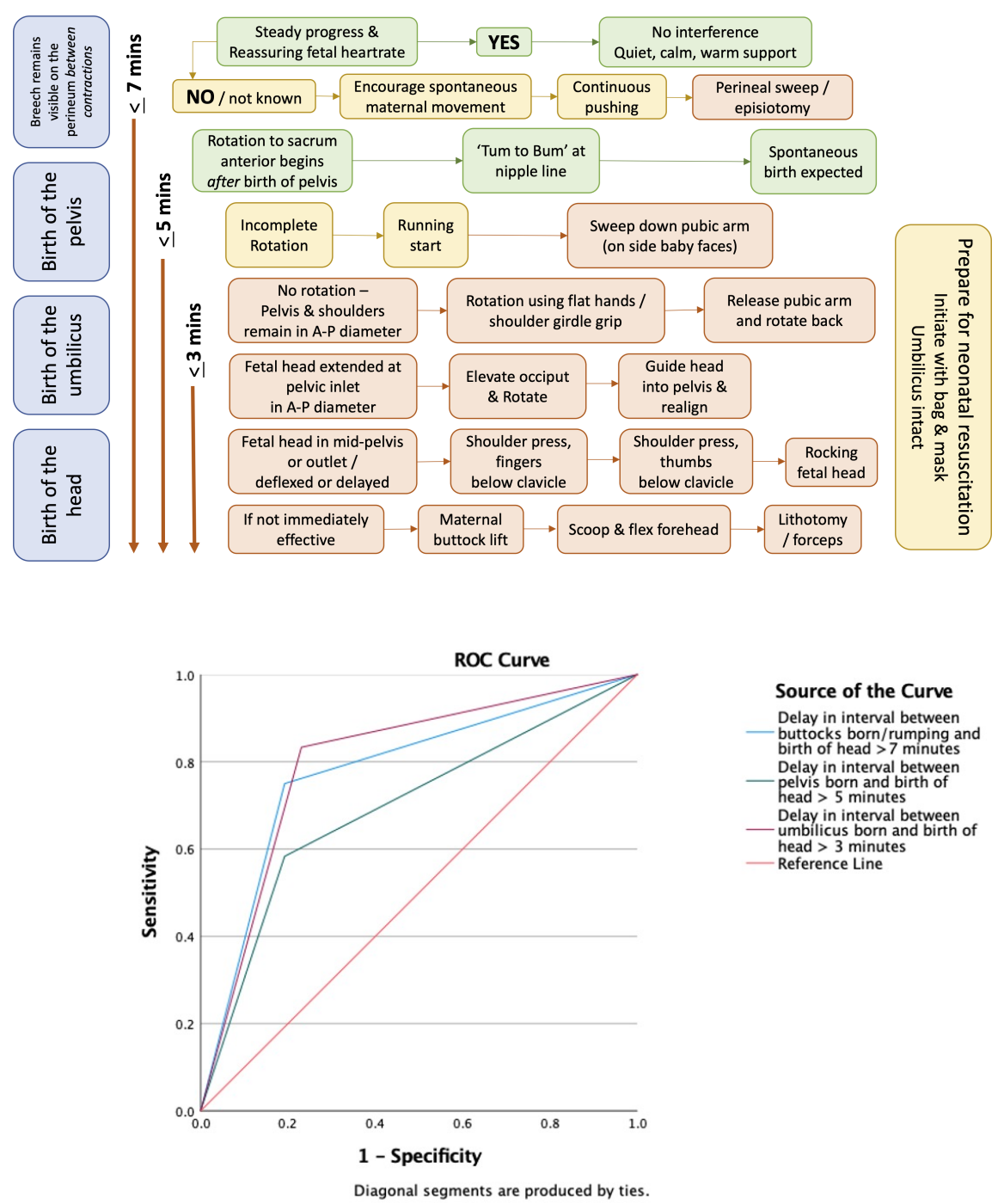

Diagonal segments are produced by ties. 